Communication

\title{
Selective Polymer Distributed Bragg Reflector Vapor Sensors
}

\author{
Paola Lova (iD \\ Dipartimento di Chimica e Chimica Industriale, Università degli Studi di Genova, Via Dodecaneso 31, \\ 16146 Genova, Italy; paola.lova@edu.unige.it
}

Received: 6 September 2018; Accepted: 14 October 2018; Published: 17 October 2018

\begin{abstract}
We report on Flory-Huggins photonic sensors for the selective detection of volatile organic compounds without the use of any chemical functionalization. For this purpose, we employed periodic multilayers made of inert cellulose acetate alternated to active polystyrene films whose free volume was modified with silanized $\mathrm{ZnO}$ nanoparticles. The simple UV-visible (UV-vis) dynamic optical response of such polymer distributed Bragg reflectors during exposure to vapors of benzene, toluene, o-dichlorobenzene, and carbon tetrachloride allows their detection and recognition based on different chemico-physical affinity with the active polymer medium.
\end{abstract}

Keywords: photonic crystals sensors; polymers; distributed Bragg reflectors; Flory-Huggins; nanoparticles

\section{Introduction}

The increasing production and consumption of goods is releasing harmful chemicals into atmosphere, water sources, and soil. Among these pollutants, volatile organic compounds (VOCs) pose serious environmental harm and concern to the human body. Table 1 provides a classification of common VOCs based on their effect on the human body. The list shows that, among others, commonly used solvents such as acetone can cause neurological damages, and that tetrachloroethylene, frequently used for dry cleaning, is a suspected carcinogenic agent [1,2]. Among other effects, indoor VOC pollution causes sick building syndrome [3], leading to eye and pulmonary irritation, headaches, loss of coordination, and nausea [4]. In the long-term, this exposure can damage the liver, kidneys, and central nervous system. VOC poisoning is also linked to the development of cancer [5]. Moreover, these pollutants contribute to the formation of the tropospheric ozone, a greenhouse gas, by reaction with oxygen sources such as nitrogen oxides and carbon monoxide [6]. The tropospheric ozone is contributing to global warming [7] and is a powerful oxidizing agent that causes respiratory illness [8], and can react with other chemicals to form new toxic pollutants [9]. VOCs are released by many industrial processes such as oil refineries, power plants, and chemical manufactures, and in urban areas by automotive vehicles, painting works, dry cleaning, refrigerators, wood burning, and photocopy machines $[3,4,10]$. The high level of toxicity and the wide spreading of these compounds make their identification and extensive monitoring in living and working environments critical to preserve people's health and to identify proper treatments in the case of poisoning. Quantitative monitoring is commonly performed by portable detectors such as colorimetric tubes, metal oxide, and infrared sensors, which are very sensitive but lack of selectivity [11-13]. Qualitative assessment requires instead time-consuming sampling, chromatography, and detection using two or more detectors combined together [14]. These characteristics make the label-free photonic sensors described here a promising alternative $[15,16]$. 
Table 1. List of common volatile organic compounds (VOCs) based on their effect on the human body.

\begin{tabular}{cl}
\hline \multicolumn{1}{c}{ Effects } & \multicolumn{1}{c}{ Compounds } \\
\hline \multirow{3}{*}{ Toxic to organ systems [1] } & $\begin{array}{l}\text { Toluene, xylenes, chlorobenzene, dichlorobenzenes, styrene, } \\
\text { carbon disulfide, acrolein, chloroform, bromoform, 2-butanone, } \\
\text { 1,3-butadiene, tetrachloroethane, dichloroethene, chloroethane, } \\
\text { dichloropropenes, bromomethan, hydrazines. }\end{array}$ \\
\hline \multirow{3}{*}{ Mutagen and developmental [1] } & $\begin{array}{l}\text { Xylenes, n-hexane, ethylene glycol, vinyl chloride, acrylonitrile, } \\
\text { acrylamide, ethylbenzene, chloroform, chloroethane, } \\
\text { dichlorobenzenes, phalates, ethylene oxide. }\end{array}$ \\
& $\begin{array}{l}\text { Fuels and mineral oils, toluene, acetone, n-hexane, benzene, } \\
\text { xylenes, acrylonitrile, pyridine, trichloroethane, carbon }\end{array}$ \\
& $\begin{array}{l}\text { tetrachloride, chloroform, 1,3-butadiene, ethylbenzene, } \\
\text { mercaptanes, naphtalenes. }\end{array}$ \\
\hline Pourological [1] & $\begin{array}{l}\text { Polycyclic aromatic hydrocarbons, acrylonitrile, acrylamide, } \\
\text { nitrobenzene, styrene, hydrazines, naphtalenes, halogenated } \\
\text { hydrocarbons (e.g., chloroform, carbon tetrachloride, } \\
\text { dichlorobenzenes, trichloroethylene, hexachloroethane } \\
\text { polyhalogenated biphenyles) }\end{array}$ \\
\hline Carcinogen [2] & Benzene, formaldehyde, vinyl chloride, ethylene oxide, \\
& benzidine, 1,3-butadiene, bis(chloromethyl) ether. \\
\hline
\end{tabular}

Recently, photonic sensors based on the Flory-Huggins interaction parameter between analytes and all-polymer distributed Bragg reflectors (DBRs) entered in the spotlight for the development of label-free colorimetric sensors capable of broad selectivity among a large amount of analytes and that do not require specific chemical functionalization [17-20]. In these systems, DBRs made of alternated layers of two polymers with different refractive index [21] play as dense membranes for the intercalation of the analytes interacting selectively with them, thus allowing detection of any kind of permeating chemical species through the simple estimation of the Flory-Huggins parameter $(\chi)$. Moreover, thanks to low fabrication costs, ease of integration in lab-on-a-chip devices and of large area production using established technologies used in the food packaging industries [22-27], these DBR sensors are very promising for the development of safety devices and quali-quantitative detection systems.

In detail, the interaction between light and DBRs provide a typical optical response recognizable as a strong peak in their reflectance spectrum, also known as photonic band-gap (PBG) [28-31]. Such a peak arises from the coherent diffraction of incident light beams occurring at all the interfaces within the multilayer. The PBG can then be compared to the X-ray diffraction peaks arising from crystalline materials. Indeed, as the variation of lattice parameters in a crystal affects its diffraction pattern, variation in periodicity and refractive index of a DBR affects its reflectance spectrum. Then, when an analyte intercalates within the multilayer, swelling or shrinking the polymer chains, the photonic lattice, and in turn its spectrum, are modified. In sensing, the different chemico-physical affinity between analytes and polymers induces different intercalation kinetics, followed by the dynamic evolution of the DBR optical response. Indeed, the intercalation kinetics are strictly related to the polymer-analytes solubility, and then to the Flory-Huggins parameter for the couples [17], which has successfully been used to describe selectivity in polymer DBRs made by the alternated layer of a block-copolymer and poly(vinyl alcohol) [20]. This allows recognition of the analyte and its concentration [15]. Such systems have been proven to be sensitive to a variety of analytes including toluene [15], water [32], alcohols, and even perfluorinated compounds [17,33].

In a previous work, we demonstrated that DBRs containing phase changing polymers, which form a semicrystalline clathrate with different optical properties through analyte intercalation, allow label free selectivity among carbon tetrachloride (CTC), benzene (BEN), toluene (TOL), and 1,2-dichlorobenzene (o-DCB) [16]. In such a case, the selectivity was achieved by exploiting 
the different kinetic of crystallization of the active polymer when exposed to the analytes, and the specific porosity imprinted by the desorbed analytes within the active sensing medium. In this work, we show that the dynamic optical response of the DBR also allows the use of amorphous commodity polymers to recognize these VOCs. To this end, we used cellulose acetate (CA, $n=1.46$ ) as low index and inert medium and a polystyrene (PS) nanocomposite doped with $\mathrm{ZnO}$ nanoparticles (ZnONP@PS, $n=1.59$ [15]) as high refractive index and active sensing medium to detect and recognize TOL, BEN, o-DCB, and CTC. Indeed, CA has large free volume and permeability to gas and vapors, and its Flory-Huggins parameter ranges from 1.2 to 2.1 for the four analytes (Table 2), making it a an appropriate inert medium [15]. Conversely, PS permeability is usually low, but its Flory-Huggins parameter is usually very small for the chemical species under consideration, making it well suitable as active medium (Table 2). To increase its permeability, we doped the dense matrix with silanized nanoparticles to introduce modification in the polymer configuration at the $\mathrm{ZnO}$-polystyrene interface [15]. This approach grants an increase of the polymer free-volume and then of the vapor permeability, allowing sensitivity below the part per million (ppm) and lower detection limit of $\sim 20 \mathrm{ppm}$ to toluene vapors [15]. This also allows faster response than those reported for bare polymer DBRs $[15,17,20]$, but while maintaining the high processability typical of amorphous polymers, which is a key factor for scaled-up fabrications. Indeed, DBRs made of amorphous commodity polymers are currently the only photonic structure availing of industrial fabrication techniques [34]. This aspect, together with their colorimetric optical response, makes these systems interesting disposable sensors for air pollutants in industrial areas, as well as integrable transductors for detection of degradation by-products in goods packaging.

\section{Materials and Methods}

Synthesis and functionalization of $\mathrm{ZnO}$ nanoparticles (NPs): ZnONPs synthesis and silanization was run accordingly to previous reports $[15,35]$. The particles were synthetized via a solvothermal route from zinc acetate dihydrate and potassium hydroxide: $0.07 \mathrm{~mol}$ of zinc acetate were dissolved in methanol and heated at $63{ }^{\circ} \mathrm{C}$ under sonication. Once this temperature was reached, $0.14 \mathrm{~mol}$ of $\mathrm{KOH}$ was dissolved in the same solvent and slowly added to acetate solution. After $3 \mathrm{~h}$ of reaction under sonication, the particles were purified by five cycles of decantation and washing with methanol, and finally dried [36]. To avoid aggregation, dimethyl-(methoxy) octadecylsilane (DMMOS) was grafted onto their surface: $10 \mathrm{~g}$ of NPs was dispersed methanol and sonicated. Then, $70 \mathrm{~mL}$ of dichloromethane containing $2.5 \mathrm{~g}$ of DMMOS was added to the NP. The new dispersion was sonicated until complete evaporation and dried in a vacuum for $2 \mathrm{~h}$. The graft reaction was then run at $135^{\circ} \mathrm{C}$ under nitrogen flux for $2 \mathrm{~h}$.

Active polymer and DBR preparation: after silanization, the ZnONPs were dispersed into a toluenepolystyrene (MW $=200,000)$ solution and stirred. The new dispersion was used to grow DBRs alternating it to cellulose acetate (MW $=61,000$ ) dissolved in 4-hydroxy-4-methylpentan-2-one (diacetone alcohol, DA). The polymer concentrations were $3 \%$ and $4 \%(w / v)$ for PS and CA, respectively. The rotation speed was 160 rounds per second.

For all the DBR samples, reflectance data were collected with home-made setups based on optical fiber using a Y-fiber probe and an Avantes (Apeldoorn, The Netherlands) AvaSpec-2048 spectrometer (200-1150 nm, resolution $1.4 \mathrm{~nm}$ ). The light source was a combined deuterium-halogen Micropak DH2000BAL (Ocean Optics, Largo, FL, USA).

Sensing measurements were performed as previously described [15] at $20^{\circ} \mathrm{C}$ and $1 \mathrm{~atm}$ with the optical set-up described above using a dip-probe Y-fiber (FDP-7UVIR200-2-yy, Avantes, Apeldoorn, The Netherlands) where the sample was placed. The probe was then inserted and sealed in a glass tube where $500 \mu \mathrm{L}$ of liquid analyte were previously placed to saturate the air environment. The distance between the sample and the liquid surface was kept at about $3 \mathrm{~cm}$. The partial pressure for the four vapors in the given condition was calculated as follows: [37] carbon tetrachloride $11.95 \mathrm{kPa}$, benzene $10.5 \mathrm{kPa}$, 1,2-dichlorobenzene $0.13 \mathrm{kPa}$, and toluene $2.8 \mathrm{kPa}$ (Table 2). 


\section{Results and Discussion}

The DBR sensors investigated in this work are made of 10 bilayers of CA and ZnONPs@PS nanocomposite supported on glass substrates. The samples appear blue, with the typical iridescence of photonic crystals (Figure 1a). In their reflectance spectrum, shown in Figure 1b, it is indeed possible to detect a maximum of intensity in the blue region of the visible spectrum at $\sim 460 \mathrm{~nm}$, which is assigned to the PBG, and provides the DBR with the blue color. In the spectrum background, an interference fringe pattern is observed, indicating the presence of well-defined external interfaces and an overall good optical quality [28-30].

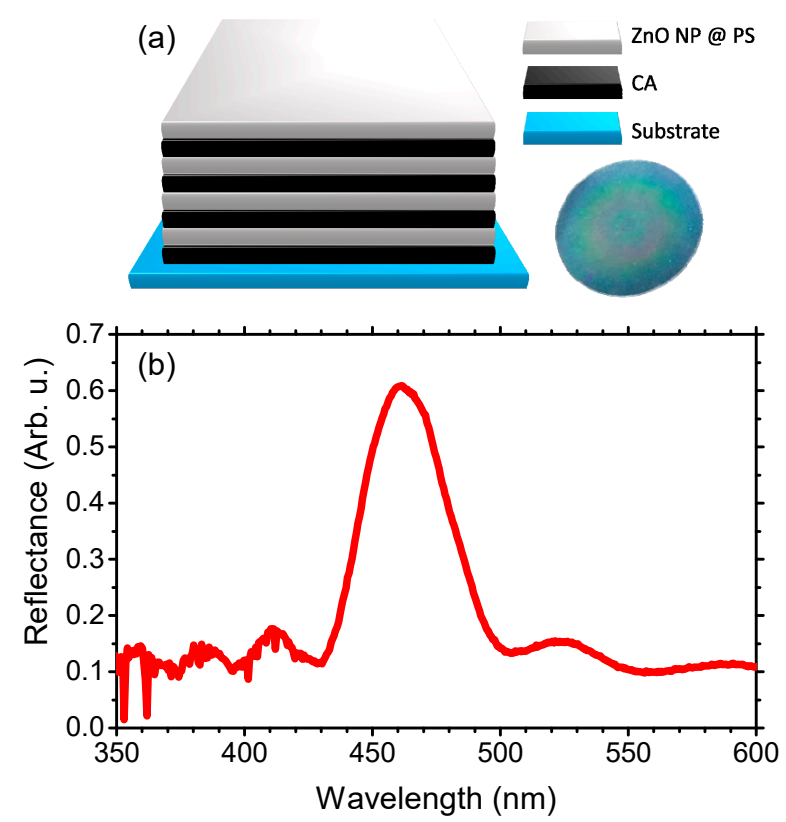

Figure 1. (a) Schematic and photograph of the ZnO nanoparticles (ZnONP)@PS/CA distributed Bragg reflector (DBR). (b) Reflectance spectrum of the sample.

The DBR sample shown in Figure 1 was cut into different portions, and each of them was exposed to saturated vapor of BEN, TOL, o-DCB, and CTC in a close environment. Figure 2 shows the dynamic optical response of the sensors measured during the exposure to the four vapor analytes. The top panels $\left(a^{\prime}-d^{\prime}\right)$ of the figure report the spectra collected before the exposure (black line) and after the equilibrium saturation is reached (red line). The bottom panels show instead the dynamic response over the entire exposure time as a contour plot $(a-d)$. Here, the $x$-scale represents the wavelengths, while the y-axes report the exposure time. The spectra intensity is instead shown as color code from blue for lower reflectance, to red for higher values, as shown in the sidebar.

Figure $2 \mathrm{a}, \mathrm{a}^{\prime}$ displays the data collected during the exposure to CTC vapors. In this case, the PBG, positioned at $460 \mathrm{~nm}$, undergoes several intensity oscillations until its intensity fades at 16 min of exposure. Contemporarily, a new peak appears at $535 \mathrm{~nm}$. This feature undergoes a monotone red-shift until $640 \mathrm{~nm}$ in $\sim 18 \mathrm{~min}$, when the response reaches the steady state. We also notice the intensity of this feature slightly fading in the time interval between $5 \mathrm{~min}$ and $15 \mathrm{~min}$. This behavior has been previously observed for polymer DBR sensors and is the result of the progressive swelling of the DBR layers from the top one in contact with the vapor rich environment [15]. Indeed, the intensity of the PBG at $460 \mathrm{~nm}$ fades as a result of the swelling of the overlaying polymer layers, which do not contribute to the formation of the PBG anymore. Such swelling affects the total thickness of the DBR, and in turn dynamically affects the interference pattern. The spectral interference between the Fabry-Perot pattern and the PBG generated the intensity and spectral oscillation detected in the PBG spectral region. The feature arising at $535 \mathrm{~nm}$ is instead assigned to the PBG of the swollen layers. It is 
indeed located at longer wavelength (larger layer thicknesses) than the original PBG and undergoes further red-shift while the layers keep swelling.

When exposed to BEN and TOL, samples show a similar behavior, but in the case of benzene, the PBG of the swollen structure is detectable after $11 \mathrm{~min}$ at $\sim 600 \mathrm{~nm}$ (Figure $2 b, b^{\prime}$ ), while for toluene, the kinetic is slower, and the PBG reaches $550 \mathrm{~nm}$ in the same amount of time, while it requires double the time of that required by BEN to reach the steady state (Figure 2c, $c^{\prime}$ ). o-DCB provides a further different response (Figure $2 \mathrm{~d}, \mathrm{~d}^{\prime}$ ). In this response, the PBG observed at $460 \mathrm{~nm}$ shows the intensity oscillation previously observed, and completely fades within $35 \mathrm{~min}$ of vapor exposure. On the other hand, the spectral feature assigned to the swollen DBR sensor cannot be detected clearly. This particular effect has been previously assigned to the large steric hindrance of this molecule, which induces severe swelling as well strain and disorder in the DBR, which break its periodicity and then hinder the formation of the PBG [16].
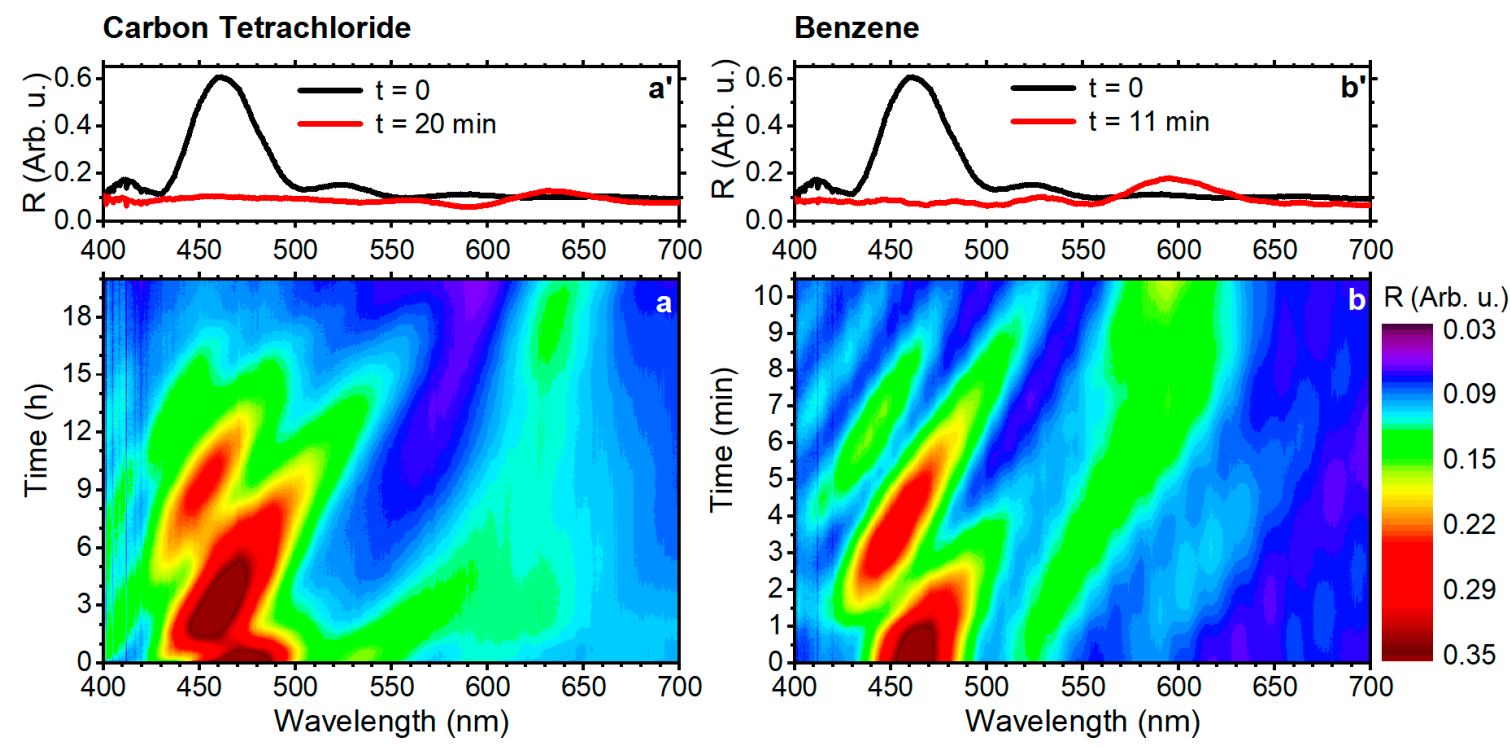

Toluene

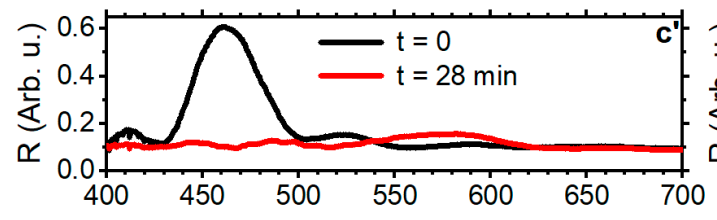

Ortho dichlorobenzene
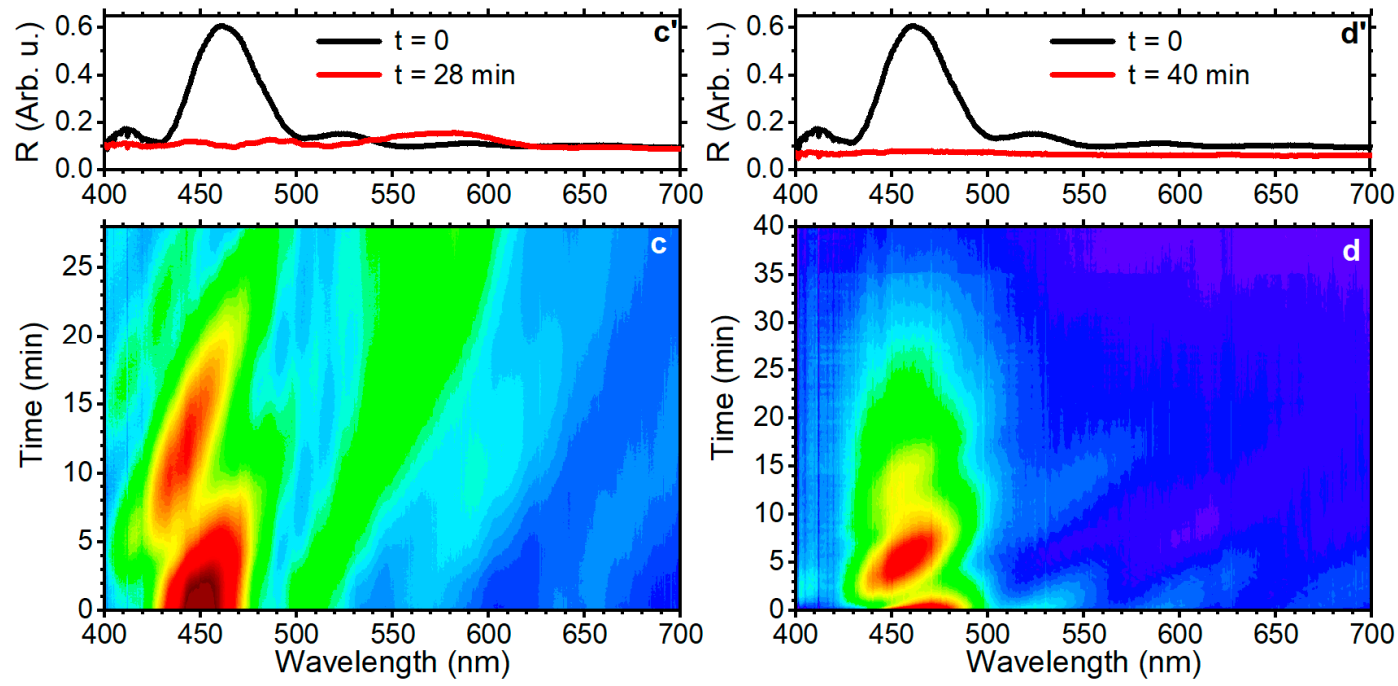

Figure 2. Optical response of the DBR sensors to vapors. The bottom panel reports the dynamic responses as contour plots $(\mathbf{a}-\mathbf{d})$. The top panels $\left(\mathbf{a}^{\prime}-\mathbf{d}^{\prime}\right)$ show the spectra collected before and after the exposures for carbon tetrachloride (CTC) $\left(\mathbf{a}, \mathbf{a}^{\prime}\right)$, benzene (BEN) $\left(\mathbf{b}, \mathbf{b}^{\prime}\right)$, toluene (TOL) $\left(\mathbf{c}, \mathbf{c}^{\prime}\right)$, and 1,2-dichlorobenzene (o-DCB) (d, $\left.\mathbf{d}^{\prime}\right)$. 
The very different optical response allows us to simply recognize the analytes. Indeed, in setting an arbitrary response time, it is possible to distinguish the analyte by the different spectral position of the PBG. To this end, Figure 3 compares the spectra of the sensors collected after $10 \mathrm{~min}$ of exposure to the vapors. Here, we notice that while the fingerprint of the response for TOL and BEN is similar (compares Figure $2 \mathrm{~b}, \mathrm{c}$ ), their very different dynamic allows their recognitions. Indeed, after $10 \mathrm{~min}$, toluene only induces a decrease in intensity of the PBG, which appears slightly blue-shifted with respect to its initial position (compare red and black lines in Figure 3). Conversely, the same exposure time allows the formation of a PBG assigned to the swollen structure when benzene is used. In this case, the PBG appears indeed at $\sim 590 \mathrm{~nm}$. CTC and o-DCB instead induce shifts of $\sim 20 \mathrm{~nm}$ and $\sim 30 \mathrm{~nm}$ respectively. These data demonstrate that the sensors response time can be as low as $10 \mathrm{~min}$. Moreover, the literature shows that engineering the DBR layer thickness and the nanoparticle volume fraction, it is also possible to achieve faster responses $[15,17,20]$. Concerning the reversibility of the sensors, a previous report on a similar system [15] demonstrated that the sensor shows full spectral reversibility upon several cycles of analyte exposure and desorption, but the response velocity increases linearly with the number of exposures. Despite the lack of full reversibility, as mentioned above, these systems are interesting disposable devices thanks to available industrial fabrications at the square-meter scale [25].

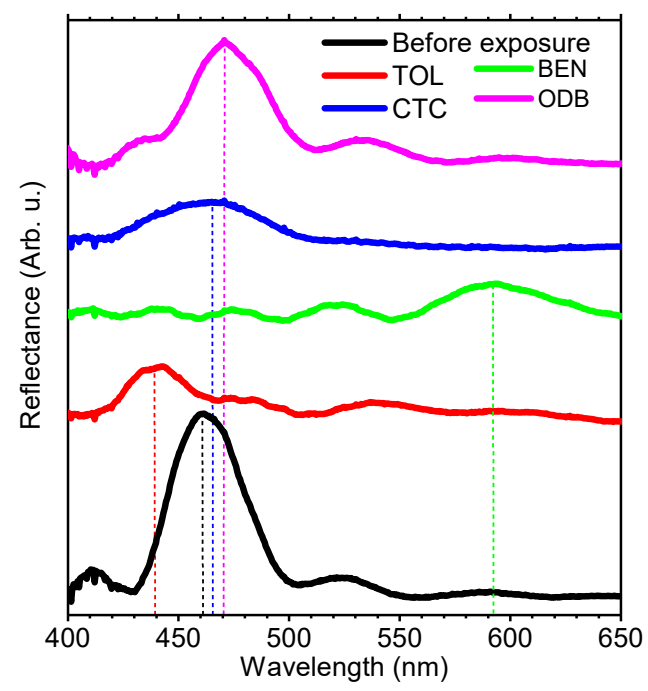

Figure 3. Reflectance spectra of ZnONP@PS @CA DBRs collected before (black line) and after $10 \mathrm{~min}$ of exposure to vapors of TOL (red), BEN (green), CTC (blue), o-DCB (magenta).

Table 2 compares the optical shift of the PBG and the time required to reach saturation retrieved for the four VOCs with their van der Waals volumes, and Flory-Huggins interaction parameters. We notice that for the benzene derivates (BEN, TOL, and o-DCB), the values of $\Delta \lambda_{\text {eq }}$ and teq increase with the analyte volumes and with $\chi_{\mathbf{P S}}$, while no correlation appears with the interaction parameter calculated for CA, confirming that the PS matrix acts as active sensing medium. No correlation between the analyte concentration and the time or spectral shift can be evinced, suggesting that the selective response is independent from these parameters. Concerning CTC, both the PBG spectral shift at saturation and saturation time do not follow the same trend, and the response time is up to 100 times slower than for benzene derivates, while the spectral shift is lower than the one induced by these molecules, which have larger van der Waals volumes. Such behavior has already been observed in the literature and can be ascribed to different intermolecular forces instauration with the two polymers with respect to the benzene derivates [16]. 
Table 2. Analytes van der Waals Volumes (V); Flory-Huggins parameter for PS ( $\left.\chi_{P S}\right), C A\left(\chi_{C A}\right)$, and for the entire DBR $\left(\chi_{\text {eff }}\right)$; photonic band-gap (PBG) spectral shift at the equilibrium $\left(\Delta \lambda_{\text {eq }}\right)$; time required to reach saturation $\left(t_{e q}\right)$; and analyte vapor pressure.

\begin{tabular}{cccccccc}
\hline Analyte & $\mathbf{V}(\mathbf{A} 2)[16]$ & $\chi_{\mathbf{P S}}[38]$ & $\chi_{\text {CA }}[38]$ & $\chi_{\text {eff }}[38]$ & $\Delta \lambda_{\text {eq }}(\mathbf{n m})$ & $\mathbf{t}_{\text {eq }}(\mathbf{m i n})$ & Vapor Pressure $(\mathbf{k P a})$ \\
\hline benzene & 89.4 & 0.001 & 1.635 & 1.183 & 130 & 11 & 10.5 \\
toluene & 106.8 & 0.006 & 2.090 & 1.512 & 120 & 28 & 2.8 \\
o-dichlorobenzene & 112.8 & 0.088 & 1.223 & 0.887 & - & 40 & 0.13 \\
$\begin{array}{c}\text { carbon } \\
\text { tetrachloride }\end{array}$ & 97.1 & 0.008 & 1.942 & 1.406 & 175 & $>1200$ & 11.95 \\
\hline
\end{tabular}

\section{Conclusions}

This work demonstrates DBR sensors made of commodity polymers with high optical responsivity and selectivity to benzene, toluene, 1,2-dichlorobenzene, and carbon tetrachloride vapor exposure. The sensitivity to vapor was achieved by increasing the overall DBR permeability using a polystyrene- $\mathrm{ZnO}$ nanocomposite, while selectivity is achieved by analyzing the dynamic optical response of the sensors, which is affected by the different chemico-physical affinity between the active sensing polymer in the DBRs and the four VOCs. Together with the possibility to fabricate polymer DBR sensors on the square-meters area, these results pave the path to new low-cost disposable devices for safety purposes to be employed in indoor and outdoor industrial environments.

Funding: European Union's Horizon 2020 research and innovation program under the Marie Sklodowska-Curie grant agreement No. 643238 (SYNCHRONICS: SupramolecularlY eNgineered arCHitectures for optoelectRonics and photONICS).

Conflicts of Interest: The author declares no conflict of interest.

\section{References}

1. Agency for Toxic Substances and Disease Registry (ATSDR). Toxicological Profiles. Available online: https: / / www.atsdr.cdc.gov/index.html (accessed on 28 July 2016).

2. Straif, K.; Cohen, A.; Samet, J. Air Pollution and Cancer; Straif, K., Cohen, A., Samet, J., Eds.; IARC Scientific Publication No. 161; International Agency or Research on Cancer: Lyon, France, 2013.

3. Guo, H.; Lee, S.C.; Chan, L.Y.; Li, W.M. Risk assessment of exposure to volatile organic compounds in different indoor environments. Environ. Res. 2004, 94, 57-66. [CrossRef]

4. Wang, S.; Ang, H.M.; Tade, M.O. Volatile organic compounds in indoor environment and photocatalytic oxidation: State of the art. Environ. Int. 2007, 33, 694-705. [CrossRef] [PubMed]

5. Phillips, M.; Gleeson, K.; Hughes, J.M.B.; Greenberg, J.; Cataneo, R.N.; Baker, L.; McVay, W.P. Volatile organic compounds in breath as markers of lung cancer: A cross-sectional study. Lancet 1999, 353, 1930-1933. [CrossRef]

6. Duan, J.; Tan, J.; Yang, L.; Wu, S.; Hao, J. Concentration, sources and ozone formation potential of volatile organic compounds (VOCs) during ozone episode in Beijing. Atmos. Res. 2008, 88, 25-35. [CrossRef]

7. Hansen, J.; Sato, M.; Ruedy, R.; Lacis, A.; Oinas, V. Global warming in the twenty-first century: An alternative scenario. Proc. Natl. Acad. Sci. USA 2000, 97, 9875-9880. [CrossRef] [PubMed]

8. Lippmann, M. Health effects of ozone a critical review. J. Air Pollut. Control Assoc. 1989, 39, $672-695$. [CrossRef]

9. Seinfeld, J.H.; Pandis, S.N. Atmospheric Chemistry and Physics: From Air Pollution to Climate Change; John Wiley \& Sons: Hoboken, NJ, USA, 2012; p. 1119.

10. Vlaanderen, J.; Straif, K.; Ruder, A.; Blair, A.; Hansen, J.; Lynge, E.; Charbotel, B.; Loomis, D.; Kauppinen, T.; Kyyronen, P.; et al. Tetrachloroethylene exposure and bladder cancer risk: A meta-analysis of dry-cleaning-worker studies. Environ. Health Perspect. 2014, 122, 661-666. [CrossRef] [PubMed]

11. Wong, J.Y.; Anderson, R.L. Non-Dispersive Infrared Gas Measurement; IFSA Publishing: Barcelona, Spain, 2012; p. 120.

12. Baur, X. International workshop 2011: "How to handle import containers safely". Int. Marit. Health 2012, 63, 140-147. [PubMed] 
13. Fine, G.F.; Cavanagh, L.M.; Afonja, A.; Binions, R. Metal oxide semi-conductor gas sensors in environmental monitoring. Sensors 2010, 10, 5469-5502. [CrossRef] [PubMed]

14. Pavia, D.L. Introduction to Organic Laboratory Techniques: A Small Scale Approach, 2nd ed.; Thomson Brooks/Cole: Belmont, CA, USA, 2005; Volume 1, p. 1021.

15. Lova, P.; Manfredi, G.; Boarino, L.; Comite, A.; Laus, M.; Patrini, M.; Marabelli, F.; Soci, C.; Comoretto, D. Polymer distributed bragg reflectors for vapor sensing. ACS Photonics 2015, 2, 537-543. [CrossRef]

16. Lova, P.; Bastianini, C.; Giusto, P.; Patrini, M.; Rizzo, P.; Guerra, G.; Iodice, M.; Soci, C.; Comoretto, D. Label-free vapor selectivity in poly(p-phenylene oxide) photonic crystal sensors. ACS Appl. Mater. Interfaces 2016, 8, 31941-31950. [CrossRef] [PubMed]

17. Lova, P.; Manfredi, G.; Comoretto, D. Advances in functional solution processed planar one-dimensional photonic crystals. Adv. Opt. Mater. 2018. [CrossRef]

18. Mönch, W.; Dehnert, J.; Prucker, O.; Rühe, J.; Zappe, H. Tunable bragg filters based on polymer swelling. Appl. Opt. 2006, 45, 4284-4290. [CrossRef] [PubMed]

19. Mönch, W.; Dehnert, J.; Jaufmann, E.; Zappe, H. Flory-huggins swelling of polymer bragg mirrors. Appl. Phys. Lett. 2006, 89, 164104. [CrossRef]

20. Gao, S.; Tang, X.; Langner, S.; Osvet, A.; Harrei $\beta$, C.; Barr, M.; Spiecker, E.; Bachmann, J.; Brabec, C.J.; Forberich, K. Time-resolved analysis of dielectric mirrors for vapor sensing. ACS Appl. Mater. Interfaces 2018. [CrossRef] [PubMed]

21. Bellingeri, M.; Chiasera, A.; Kriegel, I.; Scotognella, F. Optical properties of periodic, quasi-periodic, and disordered one-dimensional photonic structures. Opt. Mater. 2017, 72, 403-421. [CrossRef]

22. Andrews, J.H.; Aviles, M.; Crescimanno, M.; Dawson, N.J.; Mazzocco, A.; Petrus, J.B.; Singer, K.D.; Baer, E.; Song, H. Thermo-spectral study of all-polymer multilayer lasers. Opt. Mater. Express 2013, 3, 1152-1160. [CrossRef]

23. Ponting, M.; Hiltner, A.; Baer, E. Polymer nanostructures by forced assembly: Process, structure, and properties. Macromol. Symp. 2010, 294, 19-32. [CrossRef]

24. Zhou, J.; Singer, K.D.; Lott, J.; Song, H.; Wu, Y.; Andrews, J.; Baer, E.; Hiltner, A.; Weder, C. All-polymer distributed feedback and distributed bragg-reflector lasers produced by roll-to-roll layer-multiplying co-extrusion. Nonlinear Opt. Quantum Opt. 2010, 41, 59-71.

25. Hou, L.; Hou, Q.; Mo, Y.; Peng, J.; Cao, Y. All-organic flexible polymer microcavity light-emitting diodes using 3M reflective multilayer polymer mirrors. Appl. Phys. Lett. 2005, 87, 243504. [CrossRef]

26. 3M DICHROIC. Available online: https://www.3m.com/3M/en_US/company-us/all-3m-products/ /3M-Dichroic-Films-for-Architectural-Laminated-Glass / ?N=5002385+3291680356\&rt=rud (accessed on 5 October 2018).

27. Chamaleonlab. Available online: http:// chameleonlab.nl/ (accessed on 5 October 2018).

28. Lova, P.; Cortecchia, D.; Krishnamoorthy, H.N.S.; Giusto, P.; Bastianini, C.; Bruno, A.; Comoretto, D.; Soci, C. Engineering the emission of broadband 2D perovskites by polymer distributed bragg reflectors. ACS Photonics 2018, 5, 867-874. [CrossRef]

29. Manfredi, G.; Lova, P.; Di Stasio, F.; Rastogi, P.; Krahne, R.; Comoretto, D. Lasing from dot-in-rod nanocrystals in planar polymer microcavities. RSC Adv. 2018, 8, 13026-13033. [CrossRef]

30. Lova, P.; Grande, V.; Manfredi, G.; Patrin, M.; Herbst, S.; Würthner, F.; Comoretto, D. All-polymer photonic microcavities doped with perylene bisimide j-aggregates. Adv. Opt. Mater. 2017, 5, 1700523. [CrossRef]

31. Manfredi, G.; Lova, P.; Di Stasio, F.; Krahne, R.; Comoretto, D. Directional fluorescence spectral narrowing in all-polymer microcavities doped with CdSe/CdS dot-in-rod nanocrystals. ACS Photonics 2017, 4, 1761-1769. [CrossRef]

32. Manfredi, G.; Mayrhofer, C.; Kothleitner, G.; Schennach, R.; Comoretto, D. Cellulose ternary photonic crystal created by solution processing. Cellulose 2016, 23, 2853-2862. [CrossRef]

33. Giusto, P.; Lova, P.; Manfredi, G.; Gazzo, S.; Srinivasan, B.; Radice, S.V.; Comoretto, D. Colorimetric detection of perfluorinated compounds by all-polymer photonic transducers. ACS Omega 2018, 3, 7517-7522. [CrossRef]

34. Song, H.; Singer, K.; Lott, J.; Wu, Y.; Zhou, J.; Andrews, J.; Baer, E.; Hiltner, A.; Weder, C. Continuous melt processing of all-polymer distributed feedback lasers. J. Mater. Chem. 2009, 19, 7520-7524. [CrossRef] 
35. Lova, P.; Manfredi, G.; Boarino, L.; Laus, M.; Urbinati, G.; Losco, T.; Marabelli, F.; Caratto, V.; Ferretti, M.; Castellano, M.; et al. Hybrid ZnO: Polystyrene nanocomposite for all-polymer photonic crystals. Phys. Status Solidi C 2015, 12, 158-162. [CrossRef]

36. Sun, D.; Wong, M.; Sun, L.; Li, Y.; Miyatake, N.; Sue, H.-J. Purification and stabilization of colloidal ZnO nanoparticles in methanol. J. Sol-Gel Sci. Technol. 2007, 43, 237-243. [CrossRef]

37. Perry, R.H.; Green, D.W. Perry's Chemical Engineers' Handbook, 8th ed.; McGraw-Hill Professional: New York, NY, USA, 1999; Volume 1, p. 2704.

38. Hansen, C.M. Hansen Solubility Parameters: A User's Handbook, 2nd ed.; CRC Press: Boca Raton, FL, USA, 2002.

(C) 2018 by the author. Licensee MDPI, Basel, Switzerland. This article is an open access article distributed under the terms and conditions of the Creative Commons Attribution (CC BY) license (http://creativecommons.org/licenses/by/4.0/). 